\title{
Postoperative serum Troponin I elevation after thoracotomy versus video-assisted thoracoscopic lung resection
}

\author{
Morales P, Coronado C, Camio E, Matarin S, González-Tallada A, de Nadal M. \\ Department of Anaesthesiology \& Intensive Care. \\ Hospital Universitari Vall d'Hebron. Universitat Autònoma de Barcelona. Barcelona, Spain
}

Background and Goal of Study: Troponin elevation after non-cardiac surgery has been related to an increased risk of postoperative mortality (I). Troponin elevation is frequent after open lung surgery, ranging from $8,8 \%$ (I) up to $20 \%(2)$. It is believed that minimally invasive approach through video-assisted thoracoscopic surgery (VATS) is associated with a lower incidence of major cardiopulmonary complications and mortality compared to open thoracotomy (3), but there is a lack of information were it might be related with a less troponin elevation. The aims of this study were to determine the frequency of postoperative $\mathrm{Tnl}$ elevation following thoracotomy or VATS lung resection in high-risk patients and its correlation with 30-day mortality.

Patients and methods: As part of routine postoperative care for high cardiac risk patients $(\geq 65$ years or $<65$ years with known cardiovascular pathology), we measured serum high-sensitivity Troponin I (Tnl) levels (Siemens ADVIA Centaur) during the first two postoperative days in 96 consecutive patients that underwent lobectomy $(n=70)$ or lung wedge resection $(n=26)$. The Tnl cut off used to define troponin elevation was the 99th percentile provided by our laboratory ( $\mathrm{Tnl} \geq 0.04 \mathrm{ng} /$ $\mathrm{ml})$. Patients were divided in two groups depending upon surgical approach (open thoracotomy or VATS). Baseline clinical characteristics, perioperative parameters, and 30-day mortality was assessed in both groups.

Results: Of the 96 patients screened, 44 underwent open thoracotomy (TH group) and 52 VATS (VATS group). Baseline clinical characteristics and perioperative parameters were similar in both groups. Overall 30-day mortality was of $3 / 96$ patients (3.1\%). 30-day mortality was of 2/44 patients (4.5\%) in the TH group and $1 / 52$ patient $(1.9 \%)$ in the VATS group ( $\mathrm{P}$-value chi-squared 0.825) (Table I)

The overall frequency of $\mathrm{Tnl}$ elevation was of $32.3 \%$. Tnl elevation was detected in $14 / 44$ patients $(31.8 \%)$ in the TH group and in $17 / 52$ patients $(32.7 \%)$ in the VATS group (P-value chi-squared 0.927). (Table 2 )
TABLE I. Demographic and surgical parameters

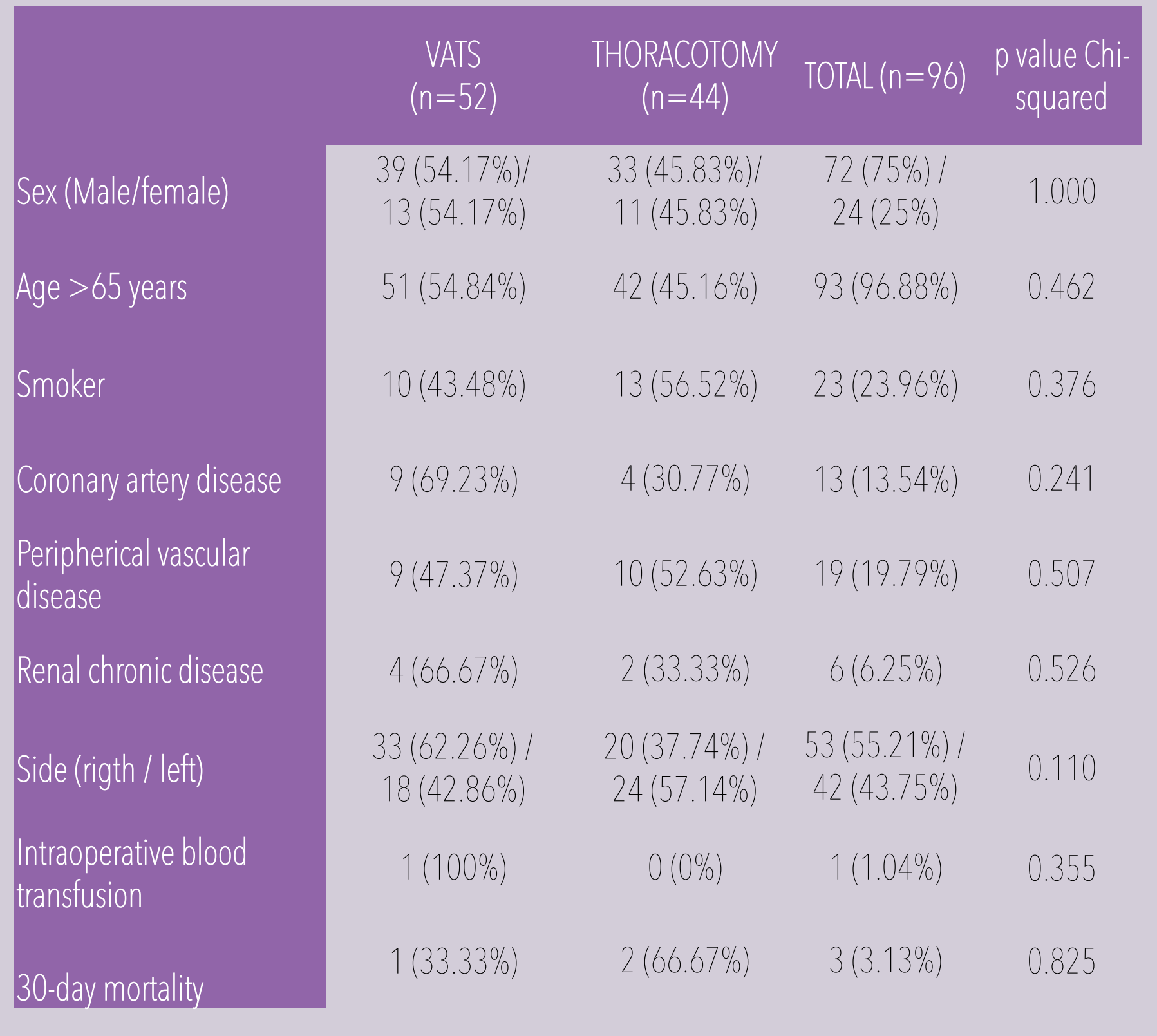

TABLE 2. Troponin elevation upon surgical approach

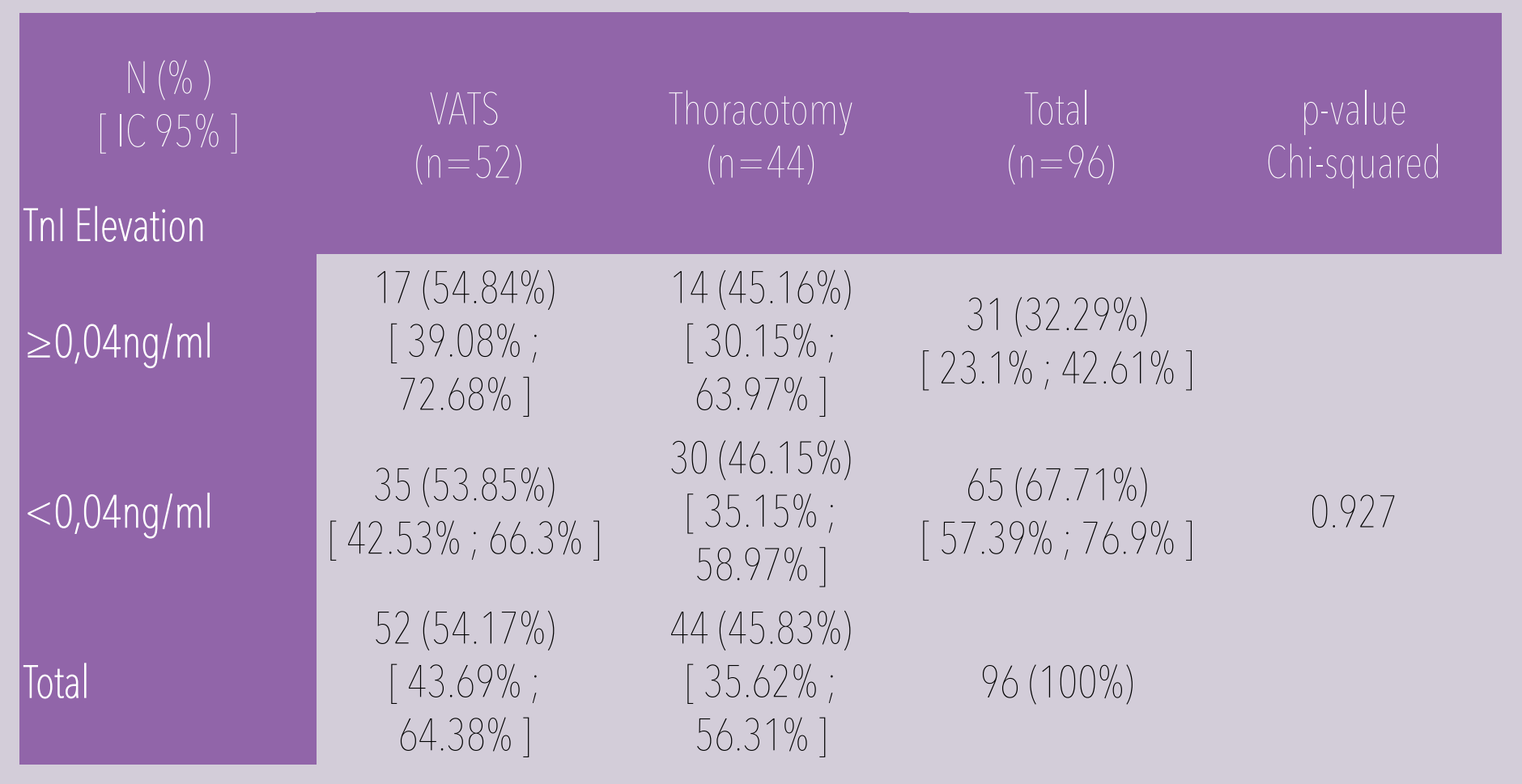

Conclusions: In high risk patients undergoing major thoracic surgery, the incidence of postoperative Tnl elevation was higher than reported in non high risk patients. Although less invasive, patients undergoing VATS showed similar troponin elevation than patients operated through open thoracotomy. No differences in 30 day mortality were observed.

References:

I. Deveraux PJ et al. JAMA. 201 2; 307:2295-2304

2. Lucrezioti $S$ et al. Rev Esp Cardiol. 2007; 60: I I 59-66

3. Falcoz PE et al. Eur J Cardiothorac Surg. 20I 5;26 pii: ezvI 54 